\title{
Detailed Dielectric Characterisation of the Heart and Great Vessels
}

\author{
Niko Ištuk*, Barry McDermott*, Emily Porter*, Adam Santorelli*, Soroush Abedi ${ }^{\dagger}$, \\ Martin O’Halloran*, Nadine Joachimowicz ${ }^{\dagger \ddagger}$, Hélène Roussel ${ }^{\S}$, \\ *Translational Medical Device Lab, National University of Ireland, Galway, Ireland, Niko.Istuk@nuigalway.ie* \\ ${ }^{\dagger}$ GeePs (CNRS - CentraleSupelec - Univ. Paris-Sud- Sorbonne Université), Gif-sur-Yvette, France \\ ‡Université de Paris, IUT, 20 Rue du Département, 75018 Paris, France \\ ${ }^{\S}$ Sorbonne Université, L2E, F-75005 Paris, France
}

\begin{abstract}
The dielectric properties of biological tissues play a significant role in the planning and development of electromagnetic thermal therapies. In most cases in the literature, heart is considered as a homogeneous organ and its dielectric properties values are reported as such. In this paper, the results of dielectric property measurements on nineteen different parts of four ovine hearts are presented. The results of the measurements indicate that the dielectric properties vary between the different parts of the heart and therefore, the heart should not be considered to be homogeneous for accurate electromagnetic modelling.
\end{abstract}

Index Terms-heart, great vessels, dielectric properties, openended coaxial probe, biological tissues, measurements, relative permittivity, conductivity

\section{INTRODUCTION}

The heart is a dual pump with carbon dioxide rich blood from the vena cavae entering the right atrium before being pumped out from the right ventricle to the lungs through the pulmonary artery [1]. Oxygen rich blood from the lungs returns through the pulmonary vein to the left atrium, before being pumped out from the left ventricle to the entire body via the aorta. This dual pump structure is shown in Fig. 1. From a histological perspective, the tissues of the heart are designed and arranged to fit a certain function. The endocardium is the inner lining of the heart, continuous with the endothelium of blood vessels [2]. Inside this layer is the cardiac muscle layer called the myocardium, which is thicker on the left side due to the need for greater force in pumping blood to the entire body as opposed to the right side that serves the lungs [2]. On the exterior surface of the heart is the epicardium, a serous membrane. Finally, a layer of fibrocartilage separates the atria and ventricles supporting the heart valves and forming openings between the chambers [2]. A previous study has also suggested that treating the heart as a homogeneous organ may not provide accurate dielectric representation of the entire organ [3].

Hence in this study, the heart was considered a heterogeneous organ with care taken to discretely measure the dielectric properties of distinct regions of both the heart and great vessels. These regions were identified based on the anatomical and functional categorisation of the heart and vessels as described above.

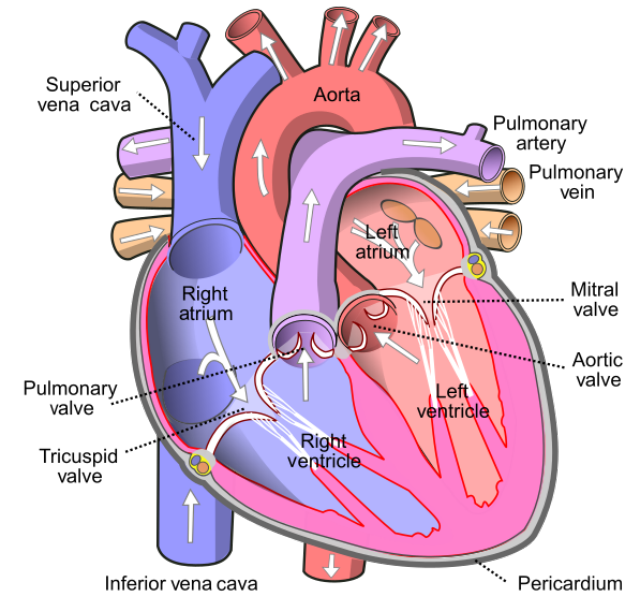

Fig. 1. Anterior (frontal) view of the opened heart [5].

\section{MATERiAls AND Methods}

\section{A. Tissue Samples}

The hearts of $n=4$ sheep were obtained from an abattoir immediately after humane slaughter using a captive bolt and carotid artery dissection. Upon death, the thoracic contents of each animal were removed, placed in an airtight container, and transported to the laboratory where the dielectric measurements were to be performed. Upon arrival in the laboratory, the hearts and great vessels were dissected away from the rest of the thoracic tissues. The time from tissue excision to first measurement was approximately 4.5 hours.

The heart can be considered a heterogeneous organ based on differing anatomy and physiology in different regions of the organ $[1,2,4]$. Further, the great vessels entering (the vena cava and pulmonary vein), and leaving (the aorta and pulmonary artery) differ in structure and function $[1,2,4]$.

We have measured the dielectric properties of the following distinct regions of both the heart ( $\mathrm{n}=15$ sites), and great vessels ( $n=4$ sites):

- Epicardium, and endocardium of the:

- Left Ventricle;

- Right Ventricle; 


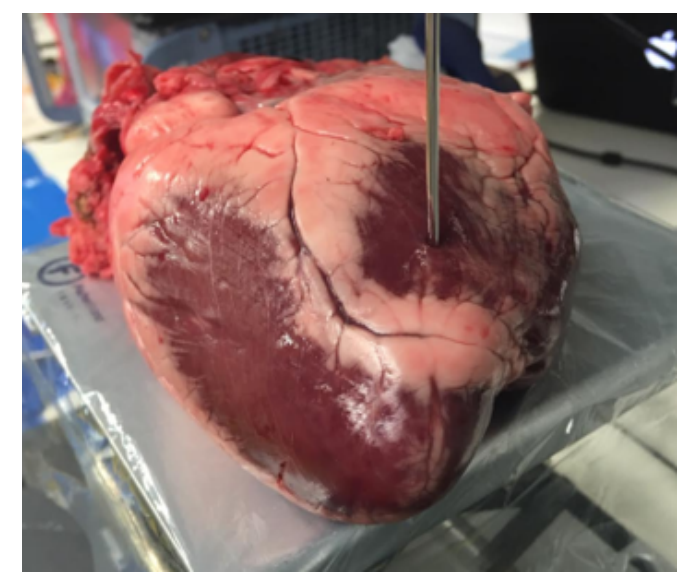

Fig. 2. Open-ended coaxial probe in contact with the ovine heart.

- Left Atrium;

- Right Atrium

- Left Atrial appendage;

- Right Atrial appendage

- Myocardium of the Septum (the dividing wall between the ventricles);

- Left Atrioventricular (mitral/ biscuspid) valve surface;

- Right Atrioventricular (tricuspid) valve surface;

- Endothelium of the Great Vessels (Aorta, Pulmonary Artery, Pulmonary Vein, Vena Cava).

\section{B. Measurement Method}

The dielectric property measurements were performed using the open-ended coaxial probe method [6,7]. Four hearts and the great vessels were measured two at a time using two open-ended coaxial probes each connected to separate vector network analyzers (VNA). One of the two slim form probes, which are a part of the $85070 \mathrm{E}$ Dielectric Probe Kit from Keysight [8], was connected to a Keysight 5063A ENA [9] VNA (Fig. 3) and the other slim form probe was connected to an Agilent (now Keysight) E8362B PNA [10] VNA. Both probes were connected directly to the VNA ports without the use of cables to avoid the measurement uncertainties that come from the cable movement [11].

In order to bring the tissue that is being measured in contact with the probe a lift table was used.

For each of the measurement locations on each of the hearts, fifteen measurements were performed. After the heart was lifted to make the contact with the probe, the first measurement was performed. After that the heart was lowered with the lift table and lifted back up to make a contact with the probe again to perform another measurement (Fig. 2).

Each time the contact of the probe with the heart was made at a slightly different position on the heart (but the same part of the heart e.g. left ventricle epicardium). This process was repeated until all of the fifteen measurements on one part of the heart were performed. After making fifteen measurements on one part of the heart, the same process was repeated for all other locations on the heart and interiors of the great vessels

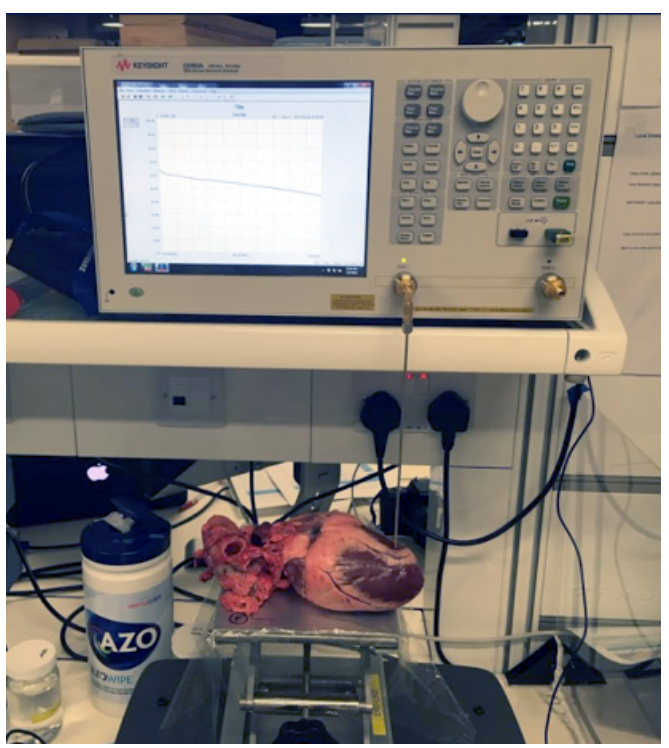

Fig. 3. Measurement setup. The heart is lifted into contact with the probe by using the lift table. The Slim form probe is directly connected with the port of the Keysight 5063A ENA.

listed above. A small longitudinal cut was made on each vessel in order to allow the probe to make the contact with the interior of the vessel.

The measurements were conducted by recording the $s_{11}$ parameter in 101 discrete frequency points with logarithmic distribution over the frequency range [12] of $200 \mathrm{MHz}$ to 8.5 $\mathrm{GHz}$ for measurements performed with the Keysight 5063A ENA and the frequency range of $200 \mathrm{MHz}$ to $20 \mathrm{GHz}$ for measurements performed with the Agilent E8362B PNA.

The measured $s_{11}$ parameters were converted to the dielectric property values by using the N1500A Materials Measurement Suite software from Keysight [13].

Both measurement setups were calibrated before the first measurements and every time 45 measurements had been performed since the last calibration (approximately every 35 minutes). The calibration method that was used is the one port calibration method [14]. The three standards used for calibration were open, short and deionized water. The validation of the calibration was done by measuring the dielectric properties of $0.9 \% \mathrm{NaCl}$ solution and comparing the measurements to the known properties from the literature [15].

The temperature of the tissue at the point of the contact with the probe was recorded for the first and for the fifteenth measurement on one measurement location using the Fluke $62 \mathrm{MAX}+$ infrared thermometer. The minimum and maximum recorded temperatures were $21.7^{\circ} \mathrm{C}$ and $25.6^{\circ} \mathrm{C}$.

The temperature of the calibration liquid (deionized water) was recorded for every calibration and it ranged from $23.5^{\circ} \mathrm{C}$ to $26.5^{\circ} \mathrm{C}$. The temperature of validation liquid $(0.9 \% \mathrm{NaCl}$ solution) was recorded for each validation and it ranged from $23.6{ }^{\circ} \mathrm{C}$ to $25.7^{\circ} \mathrm{C}$. The temperatures of both liquids were measured using the DTM 3000 digital thermometer.

The time of death of the animals and the time of excision of 


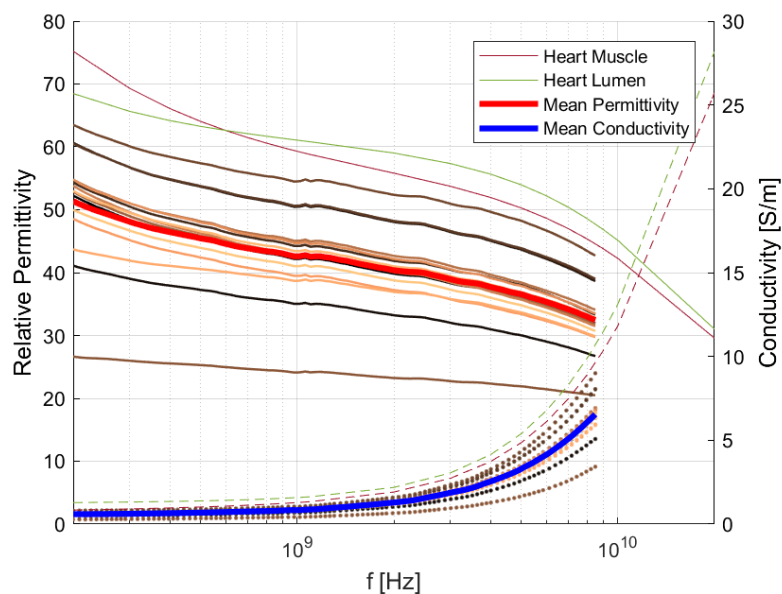

(a) left atrial appendage epicardium

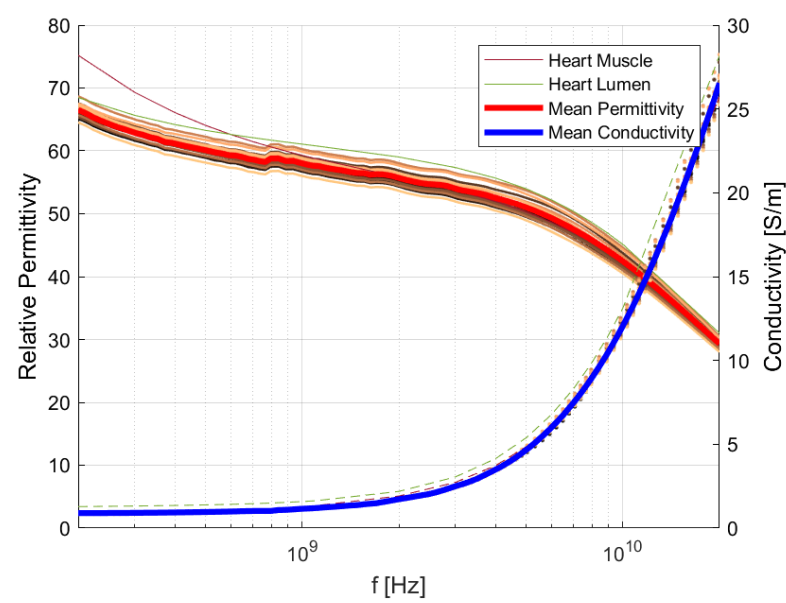

(c) interior of the pulmonary artery

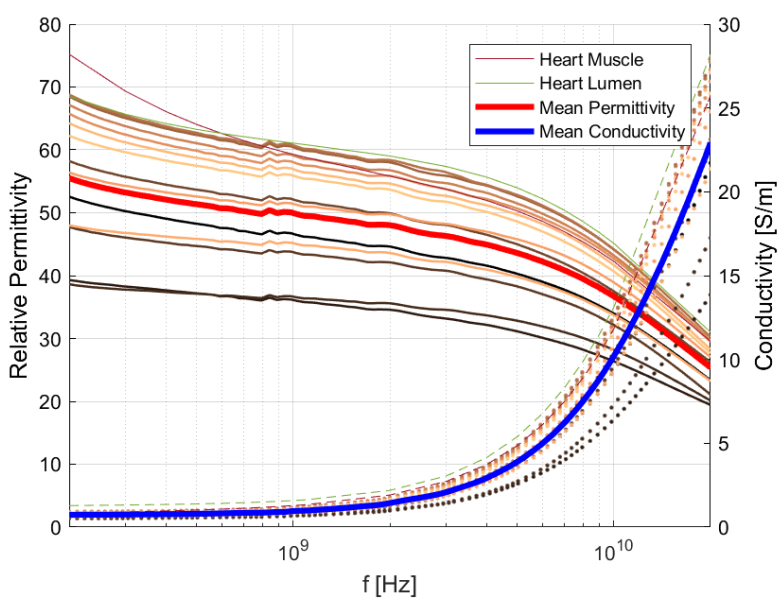

(b) left atrium epicardium

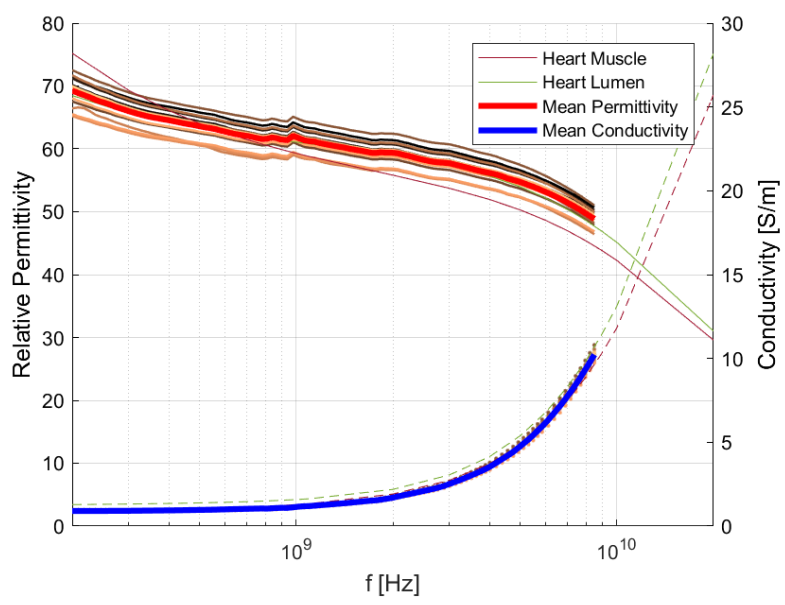

(d) right ventricle endocardium

Fig. 4. Sample of the results of the dielectric properties of the heart and the great vessels measurements. Each plot is showing fifteen measurement results for one measurement location on one of the hearts. Thick red line is the mean of the measured relative permittivity. The thick blue line is the mean of the measured conductivity. The dark red line is heart muscle and green is heart lumen from the literature $[11,18,19]$ since data from these specific regions is not available.

the heart were recorded. The time of each measurement was also recorded in order to calculate the time between the death of the animal and the measurement and the time between the excision of the heart and the measurement. All measurement were taken within 4 hours, between 4:38 and 8:39 hours after the excision.

\section{Results AND Discussion}

\section{A. Validation}

The validation measurements were done before making the first measurement on each measurement location for each of the hearts. One validation was also done after every calibration. The total number of validations is 25 for each of the hearts or 100 in total.

The validation error was calculated for each validation as the mean value across the frequency range of the percentage difference between measured values of the $0.9 \% \mathrm{NaCl}$ solution and the values from the literature [15] for each frequency. This
TABLE I

THE AVERAGE AND WORST-CASE MEAN PERCENTAGE VALIDATION ERRORS

\begin{tabular}{cccc}
\hline \multicolumn{2}{c}{ Average } & \multicolumn{2}{c}{ Worst case } \\
\hline Relative & & Relative \\
permittivity & Conductivity & permittivity & Conductivity \\
1.39 & 3.40 & 2.44 & 5.66 \\
\hline
\end{tabular}

validation error was calculated for both relative permittivity and conductivity. Worst case and average validation error are reported in Table I.

The data and metadata was collected in line with the MINDER framework [16].

\section{B. Dielectric properties of the heart and the great vessels}

In Fig. 4 four sets of fifteen measurements are shown, each on different part of a heart. 


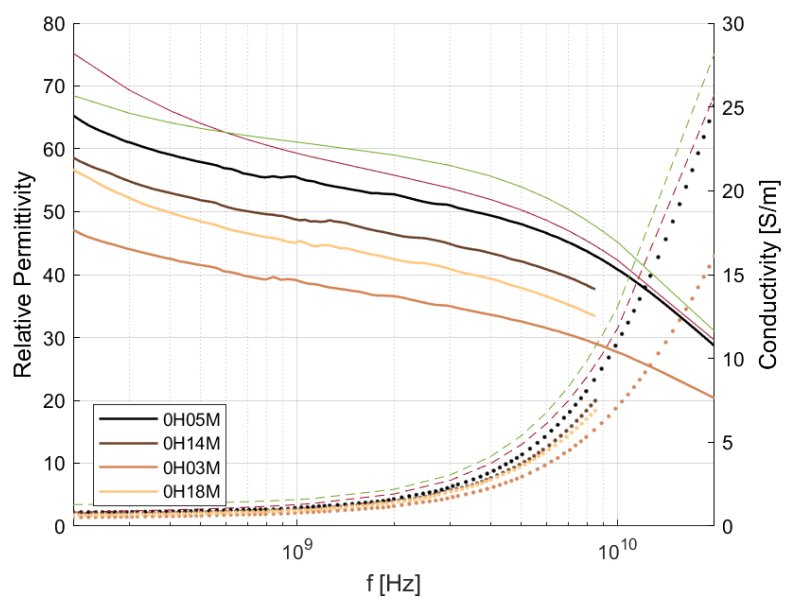

(a) left ventricle epicardium

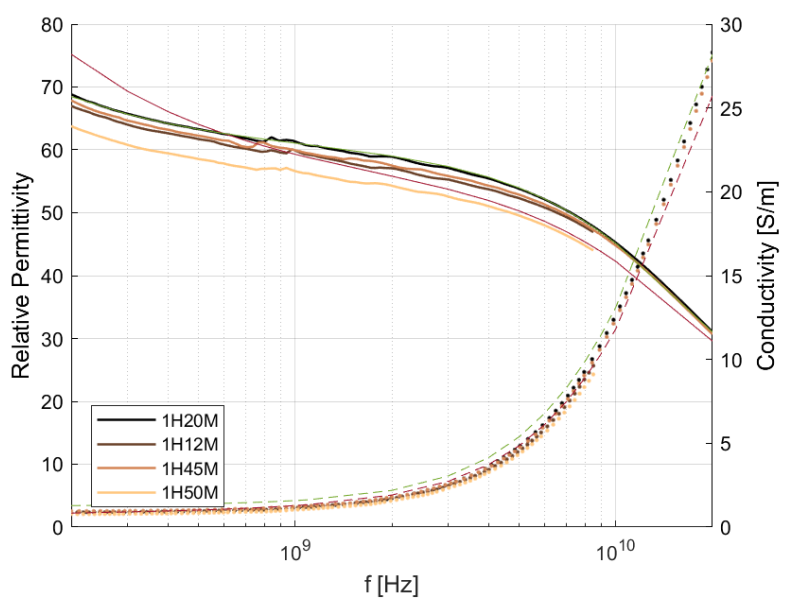

(b) left ventricle endocardium

Fig. 5. Means of the dielectric properties of the heart and the great vessels measurements for each heart. Legend shows the time (in hours and minutes) since the first measurement on the heart. Data from literature $[11,18,19]$ for heart muscle (dark red) and heart lumen (green) is plotted for comparison.

In Fig. 4a we can see the results for dielectric properties measured on the outside of the left atrial appendage of the heart number two. In Fig. 4b the dielectric properties measured on the epicardium tissue on the left atrium of the heart number four are plotted. These two sets of measurements exemplify how difficult it is to perform a consistent measurement on the outside of the heart. This can be due to the heterogeneity of the outside tissues of the heart [17]. As it can be seen on Fig. 2, on the outside of the heart, the heart muscle tissue is partially exposed and partially covered by a layer of fat tissue. Another reason for high variability of the dielectric properties measurements on the outside of the heart could be the fact that the excised tissues dehydrate over time and dehydration of the tissue changes the dielectric properties of the tissue [20].

In Fig. 4c and Fig. 4d the results of measurements on interior of the pulmonary artery of the heart number three and right ventricle endocardium of the heart number two are shown. There is much less variability in the measurements compared to Fig. 4a and Fig. 4b. This could be due to the fact that the interior of the heart is much more homogeneous compared to the outside. Also, the internal part of the excised hearts do not dehydrate as much as the outer parts as they are only exposed to the air right before the measurements are conducted.

Fig. 5 shows the mean values for the epicardium and endocardium on the left ventricle of all of the hearts. Time that has elapsed from the first measurement on each of the heart is indicated on the labels. This is the time since the hearts were taken out of the sealed airtight containers in order to start doing the measurements. This time is important because it can be considered that that is the time at which the tissue has started to dehydrate more rapidly than when it was still in the sealed container.

Fig. 5 confirms that the measurements on the outside parts of the heart, which in this case is the epicardium, show higher variability that the measurements on the inside, in this case

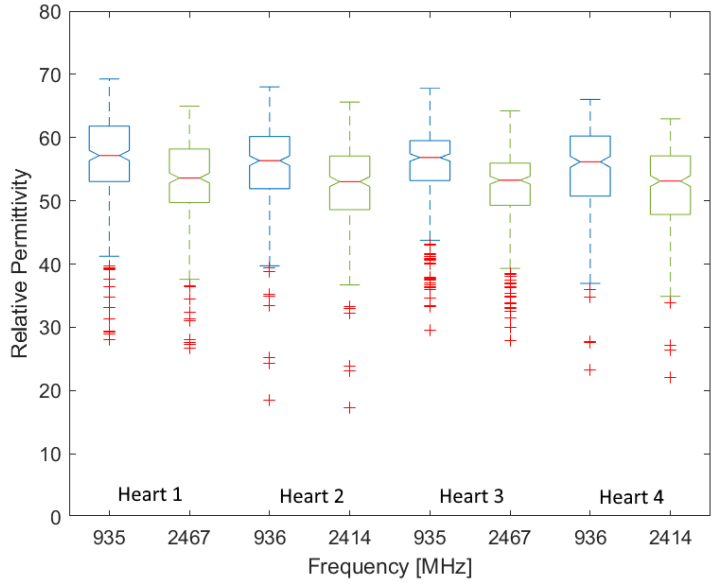

Fig. 6. Box plot for mean relative permittivity values for four different hearts at two sets of close frequencies. The mean values are calculated from all the measurements that were performed on each heart.

the endocardium.

Fig. 6 shows box plots of all of the measurements on each heart for two sets of discrete frequencies. One set of frequencies is as close to $915 \mathrm{MHz}$ as can be found in our data and the other is aimed to be close to $2.45 \mathrm{GHz}$. These frequencies were selected as they are typical frequencies in microwave frequency ablation (MFA) [3], [21]. The frequencies are slightly different due to the fact that none of the frequency points in our experiments is at that exact frequency due to the logarithmic spacing.

We can see that the results of measurements on all the hearts produce similar box plots. This tells us that there is no obvious inter-individual variability between the four organs. It is also worth noticing that all the outlier points are located bellow the boxes in the plot. This can be because the outliers may be caused either by measuring the fat tissue, which has a lower relative permittivity than the muscle tissue or by measured 


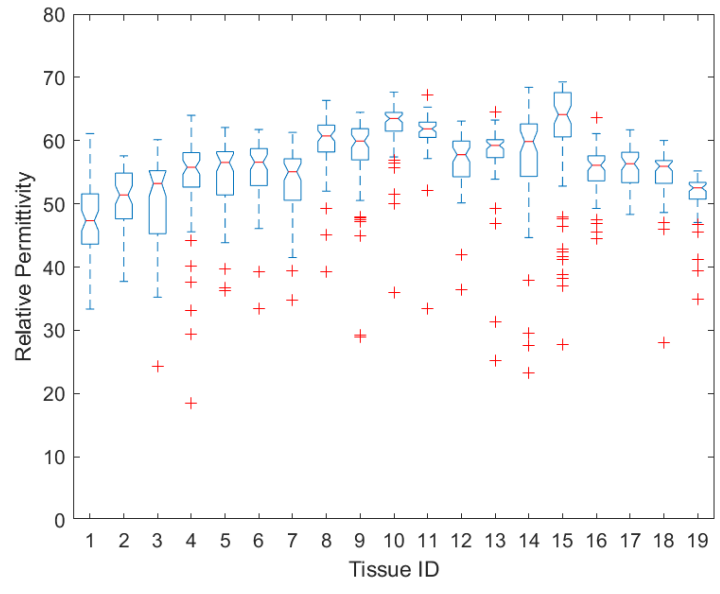

Fig. 7. Box plot for mean relative permittivity values across all four hearts for nineteen different measurement locations at $935 \mathrm{MHz}$.

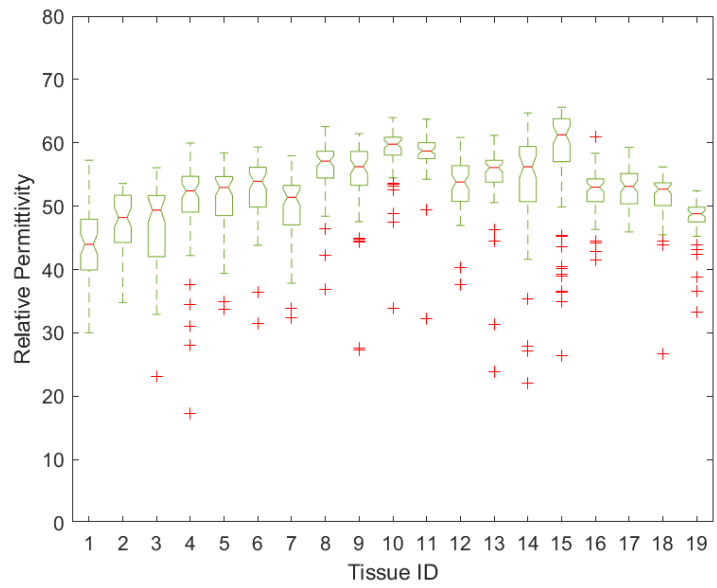

Fig. 8. Box plot for mean relative permittivity values across all four hearts for nineteen different measurement locations at $2.4 \mathrm{GHz}$.

tissues dehydration, which also leads to lower properties.

In Fig. 7 and Fig. 8 the box plots for each location of the measurement is showing more variability at both frequencies. The location of the measurement is indicated by the tissue identification number. Tissue identification numbers from 16 are epicardium tissues, 7 is septum myocardium, 8-13 are endocardium tissues, $14-15$ are the valves and 16-19 are the great vessels. This variability is an indicator that the dielectric properties vary between the different parts of the heart.

\section{CONCLUSiON}

In this paper the results of the dielectric properties measurements on different parts of four ovine hearts indicate that different parts of the heart have different dielectric properties. This is in agreement with the previous work on similar topic. We also demonstrate how some of the confounding factors in the dielectric properties measurement process, such as tissue heterogeneity and tissue dehydration, can influence the results of the measurement. Inter-individual variability, which can also be considered to be a confounding factor in the measurement process, did not play a significant role in our experiments.

\section{ACKNOWLEDGMENT}

This work has been developed in the framework of COST Action MyWAVE (CA17115) and was supported by the EMERALD project funded from the European Union's Horizon 2020 research and innovation programme under the Marie Skłodowska-Curie grant agreement No. 764479.

\section{REFERENCES}

[1] S. Standring, N. Ananad, and H. Gray, Eds., "Gray's anatomy: the anatomical basis of clinical practice, 41. ed." Philadelphia, Pa.: Elsevier, 2016.

[2] M. H. Ross and W. Pawlina, "Histology: A Text and Atlas: With Correlated Cell and Molecular Biology, Seventh edition", Philadelphia: Wolters Kluwer Health, 2016.

[3] S. Salahuddin et al., "Demonstration of dielectric heterogeneity of previously assumed homogeneous tissues: examination of the Heart," in 12th European Conference on Antennas and Propagation (EuCAP 2018), 2018.

[4] B. Singh and K. M. Dyce, Eds., "Dyce, Sack, and Wensing's Textbook of Veterinary Anatomy, 5th Edition", St. Louis, Missouri: Saunders, 2018.

[5] Eric Pierce, 02 June 2006, Diagram of the human heart (cropped), 07 October 2019, Wapcaplet, https://creativecommons.org/licenses/bysa/3.0/legalcode

[6] T. P. Marsland and S. Evans, "Dielectric measurements with an openended coaxial probe," IEE Proceedings H (Microwaves, Antennas and Propagation), vol. 134, no. 4, pp. 341-349, Aug. 1987.

[7] A. La Gioia et al., "Open-Ended Coaxial Probe Technique for Dielectric Measurement of Biological Tissues: Challenges and Common Practices," Diagnostics, vol. 8, no. 2, p. 40, Jun. 2018.

[8] Keysight 85070E Dielectric Probe Kit $200 \mathrm{MHz}$ to $50 \mathrm{GHz}$; Keysight Technologies: Santa Clara, CA, USA, 2017.

[9] E5063A ENA Vector Network Analyzer; Keysight Technologies: Santa Clara, CA, USA, 2019.

[10] Agilent PNA Microwave Network Analyzers Data Sheet; Agilent Technologies, Inc.: Santa Clara, CA, USA, 2009.

[11] A. Peyman, S. Holden, and C. Gabriel, "Dielectric Properties of Tissues at Microwave Frequencies," RUM3, Dec. 2009.

[12] S. Salahuddin, E. Porter, P. M. Meaney, and M. O'Halloran, "Effect of logarithmic and linear frequency scales on parametric modelling of tissue dielectric data," Biomed. Phys. Eng. Express, vol. 3, no. 1, p. 015020, Feb. 2017.

[13] N1500A Materials Measurement Suite Technical Overview; Keysight Technologies: Santa Clara, CA, USA, 2018.

[14] V. Teppati, A. Ferrero, and M. Sayed, Modern RF and microwave measurement techniques. Cambridge University Press, pp. 198-199, 2013.

[15] A. Peyman, C. Gabriel, and E. H. Grant, "Complex permittivity of sodium chloride solutions at microwave frequencies," Bioelectromagnetics, vol. 28, no. 4, pp. 264-274, 2007.

[16] E. Porter et al., "Minimum information for dielectric measurements of biological tissues (MINDER): A framework for repeatable and reusable data,' International Journal of RF and Microwave Computer-Aided Engineering, vol. 28, no. 3, p. e21201, Nov. 2017.

[17] E. Porter, A. L. Gioia, M. A. Elahi, and M. O'Halloran, "Significance of heterogeneities in accurate dielectric measurements of biological tissues," in 2017 XXXIInd General Assembly and Scientific Symposium of the International Union of Radio Science (URSI GASS), 2017, pp. 1-4.

[18] IT'IS Foundation, "Database: Dielectric Properties," http://www.itis.ethz.ch/itis-for-health/tissue properties/database/dielectricproperties/, 07 October 2019.

[19] IFAC, "Dielectric Properties of Body Tissues in the Frequency Range 10 Hz-100 GHz," http://niremf.ifac.cnr.it/tissprop/, 07 October 2019.

[20] A. Shahzad, S. Khan, M. Jones, R. Dwyer, and M. O'Halloran, "Investigation of the effect of dehydration on tissue dielectric properties in ex-vivo measurements," Biomedical Physics and Engineering Express, vol. 3, May 2017.

[21] C. L. Brace, "Radiofrequency and Microwave Ablation of the Liver, Lung, Kidney, and Bone: What Are the Differences?," Current Problems in Diagnostic Radiology, vol. 38, no. 3, pp. 135-143, May 2009. 